\title{
Global strict solutions to continuous coagulation-fragmentation equations with strong fragmentation
}

\author{
Jacek Banasiak \\ School of Mathematical Sciences, University of KwaZulu-Natal \& \\ Institute of Mathematics, Technical University of Lódź \\ e-mail:banasiak@ukzn.ac.za \\ and \\ Wilson Lamb \\ Department of Mathematics and Statistics, University of Strathclyde \\ e-mail:wl@maths.strath.ac.uk
}

\begin{abstract}
In this paper we give an elementary proof of the unique, global-in-time solvability of the coagulation-(multiple) fragmentation equation with polynomially bounded fragmentation and particle production rates and a bounded coagulation rate. The proof relies on a new result concerning domain invariance for the fragmentation semigroup which is based on a simple monotonicity argument.
\end{abstract}

Key words: semigroups of operators, semilinear Cauchy problem, strong solutions, coagulation, fragmentation.

AMS subject classifications: 35F25, 47D06, 45K05, 80A30.

Acknowledgement: The work presented here was completed when Professor Banasiak was a Sir David Anderson Fellow at the University of Strathclyde. Professor Banasiak is grateful for the financial support provided by the Sir David Anderson Bequest which enabled his visit to Strathclyde to take place and for support provided by the National Research Foundation of South Africa, grant GUN 2025057.

The authors thank the referee for pointing out a simplification of the estimate in Lemma 4.2. 


\section{Introduction}

In this paper we consider the continuous coagulation - (multiple) fragmentation (CF) equation

$$
u_{t}(x, t)=(\mathcal{F} u)(x, t)+(\mathcal{N} u)(x, t)
$$

where

$$
(\mathcal{F} u)(x, t)=-a(x) u(x, t)+\int_{x}^{\infty} b(x \mid y) a(y) u(y, t) d y
$$

and

$$
(\mathcal{N} u)(x, t)=\frac{1}{2} \int_{0}^{x} k(x-y, y) u(x-y, t) u(y, t) d y-u(x, t) \int_{0}^{\infty} k(x, y) u(y, t) d y
$$

model fragmentation and coagulation, respectively. The various terms appearing in (1.2) and (1.3) are interpreted in the usual manner; see [3, Chapter 8] and [12]. Thus $a(x)$ is the rate of fragmentation of a particle of mass $x, b(x \mid y)$ is a non-negative measurable function which describes the distribution of mass $x$ (daughter) particles spawned by the fragmentation of a particle of mass $y$ and $k(x, y)$ represents the rate at which particles of mass $x$ coalesce with those of mass $y$. In any fragmentation event, the average number and mass of the daughter particles formed are given, respectively, by

$$
n(y):=\int_{0}^{y} b(x \mid y) d x \quad \text { and } \quad m(y):=\int_{0}^{y} x b(x \mid y) d x .
$$

Throughout we make the following assumptions on $a, b$ and $k$.

(A.1) $a$ is non-negative, non-zero and bounded at 0 ;

(A.2) $m(y)=\int_{0}^{y} x b(x \mid y) d x=y$, for all $y>0$;

(A.3) $n(y)=\int_{0}^{y} b(x \mid y) d x \geq 1$ for all $y>0$, and $n \in L_{\infty, l o c}([0, \infty))$;

(A.4) $k$ is non-negative, symmetric and $k \in L_{\infty}\left(\mathbb{R}_{+} \times \mathbb{R}_{+}\right)$.

Other conditions will be imposed when necessary. Note that (A.2) expresses the property that mass is conserved in any fragmentation event, while the first part of (A.3) reflects the fact that there is no loss of particles when fragmentation occurs.

The approach we adopt to establish existence and uniqueness results for the CF equation involves the application of perturbation methods from the theory of semigroups of operators. The initialvalue problem associated with equation (1.1) is expressed as a semilinear abstract Cauchy problem (ACP) of the form

$$
u_{t}(t)=G u(t)+N u(t), \quad u(0)=\stackrel{\circ}{u},
$$


where $G$ is an operator realisation of the linear fragmentation process that generates a strongly continuous semigroup, $\left\{S_{G}(t)\right\}_{t \geq 0}$, and the nonlinear coagulation operator $N$ is given by

$$
(N f)(x):=\frac{1}{2} \int_{0}^{x} k(x-y, y) f(x-y) f(y) d y-f(x) \int_{0}^{\infty} k(x, y) f(y) d y .
$$

In the absence of coagulation $(k(x, y) \equiv 0)$, the pure fragmentation ACP is normally studied in the 'mass' space $X_{1}:=L_{1}\left(\mathbb{R}_{+}, x d x\right)$. In this case, it follows from (A.1) and [3, Theorem 8.5] that we can choose $G$ to be the closure of the operator $(A+B, D(A))$, where

$$
(A f)(x):=-a(x) f(x),(B f)(x):=\int_{x}^{\infty} b(x \mid y) a(y) f(y) d y, D(A):=\left\{f \in X_{1}: a f \in X_{1}\right\} .
$$

The resulting semigroup $\left\{S_{G}(t)\right\}_{t \geq 0}$ is then a strongly continuous positive semigroup of contractions on $X_{1}$.

Unfortunately, the operator $N$ does not behave well in $X_{1}$ and so an analysis of (1.5) (with $N \neq 0$ ) is usually carried out in the Banach space $X_{0,1}:=L_{1}\left(\mathbb{R}_{+},(1+x) d x\right)$ in which both the total mass and number of particles are controlled. A consequence of this is that a restriction, say $G_{0,1}$, of the generator $G$ of the fragmentation semigroup must be found such that $G_{0,1}$ generates a strongly continuous semigroup on $X_{0,1}$. This, so far, has only been possible by imposing rather severe constraints on $a$, such as linear boundedness [12]. One of our main aims here is to establish the existence and uniqueness of a solution to (1.5) for a wider class of fragmentation rates $a$.

In the sequel it will be convenient to work with the following more general versions of the spaces $X_{1}$ and $X_{0,1}$. For each $\alpha \geq 0$ and $\beta \geq 0$, let

$$
X_{\alpha}:=L_{1}\left(\mathbb{R}_{+}, x^{\alpha} d x\right) \text { and } X_{\alpha, \beta}=X_{\alpha} \cap X_{\beta}:=L_{1}\left(\mathbb{R}_{+},\left(x^{\alpha}+x^{\beta}\right) d x\right) .
$$

The norms on $X_{\alpha}$ and $X_{\alpha, \beta}$ will be denoted by $\|\cdot\|_{\alpha}$ and $\|\cdot\|_{\alpha, \beta}$ respectively. Therefore

$$
\|f\|_{\alpha}:=\int_{0}^{\infty} x^{\alpha}|f(x)| d x\left(f \in X_{\alpha}\right), \quad\|f\|_{\alpha, \beta}:=\int_{0}^{\infty}\left(x^{\alpha}+x^{\beta}\right)|f(x)| d x\left(f \in X_{\alpha, \beta}\right) .
$$

Note that

$$
X_{\alpha, \beta_{2}} \hookrightarrow X_{\alpha, \beta_{1}} \text { with }\|f\|_{\alpha, \beta_{1}} \leq 2\|f\|_{\alpha, \beta_{2}}
$$

for any $f \in X_{\alpha, \beta_{2}}$ whenever $0 \leq \alpha \leq \beta_{1} \leq \beta_{2}$. This follows since

$$
\begin{aligned}
\|f\|_{\alpha, \beta_{1}} & =\int_{0}^{\infty} x^{\alpha}|f(x)| d x+\int_{0}^{1} x^{\beta_{1}}|f(x)| d x+\int_{1}^{\infty} x^{\beta_{1}}|f(x)| d x \\
& \leq \int_{0}^{\infty} x^{\alpha}|f(x)| d x+\int_{0}^{1} x^{\alpha}|f(x)| d x+\int_{1}^{\infty} x^{\beta_{2}}|f(x)| d x \leq 2\|f\|_{\alpha, \beta_{2}} .
\end{aligned}
$$

The above also shows that, under the same conditions on $\alpha, \beta_{1}$ and $\beta_{2}$,

$$
\|f\|_{\beta_{1}} \leq\|f\|_{\alpha, \beta_{2}} \quad \forall f \in X_{\alpha, \beta_{2}}
$$


which, in turn, leads to

$$
X_{\alpha, \beta_{2}} \hookrightarrow X_{\beta_{1}, \beta_{2}} \text { with }\|f\|_{\beta_{1}, \beta_{2}} \leq 2\|f\|_{\alpha, \beta_{2}} \forall f \in X_{\alpha, \beta_{2}} .
$$

We can now state the two main results that are established in this paper. The first, Theorem 1.1, expresses an invariance property of the fragmentation semigroup $\left\{S_{G}(t)\right\}_{t \geq 0}$, and is proved in Section 3. The second, Theorem 1.2, showing that unique solutions to (1.5) exist for a wide class of fragmentation kernels $a$ and $b$ and suitably restricted $\stackrel{\circ}{u}$, is proved in Section 4 .

Theorem 1.1 Let $a$ and $b$ satisfy (A.1)-(A.3) and let a be eventually non-decreasing. Then

(a) $D(A)$ is invariant under the semigroup $\left\{S_{G}(t)\right\}_{t \geq 0}$;

(b) $X_{0,1} \cap D(A)$ is invariant under the semigroup $\left\{S_{G}(t)\right\}_{t \geq 0}$ under the following additional assumption on $b$ :

$$
\int_{0}^{y} b(x \mid y) d x=n(y) \leq c_{1}+c_{2} y \text { for some } c_{1} \geq 1 \text { and } c_{2} \geq 0 .
$$

Part (a) extends an earlier result by the first author [1] for binary fragmentation in which invariance of $D(A)$ is established for the case $a(x)=x^{\alpha}, \alpha>0$ and $b(x \mid y)=2 / y$. The significance of (a) is that it provides sufficient conditions for $u(t)=S_{G}(t) \stackrel{\circ}{u}$ to be a strict solution, not only of $u_{t}(t)=G u(t)$, but also of $u_{t}(t)=A u(t)+B u(t)$. Part (b) shows that the number of particles, as well as the mass, can be controlled if $n$ is assumed to be linearly bounded. This result is clearly of relevance to the $\mathrm{ACP}(1.5)$ as the invariance result stated relates to the space $X_{0,1}$.

Theorem 1.2 In addition to (A.1)-(A.4), assume that $b$ satisfies

$$
\int_{0}^{y} b(x \mid y) d x=n(y) \leq c_{1}+c_{2} y^{r} \text { for some } c_{1} \geq 1, r \geq 1 \text {, and } c_{2} \geq 0,
$$

and that constants $C>0$ and $p \in(1, \infty)$ exist such that

$$
a(x) \leq C\left(1+x^{p}\right) \quad \forall x \geq 0
$$

Then, for $m=p+r$ and $0 \leq \stackrel{\circ}{u} \in X_{0, m}$ there exists a unique, globally-defined, non-negative mild solution $u:[0, \infty) \rightarrow X_{0, m}$ of the semilinear ACP (1.5).

In essence, Theorem 1.2 shows that a global solution to (1.5) can be found when $a$ and $n$ are polynomially bounded provided that sufficiently high moments of the initial condition $\stackrel{\circ}{u}$ exist. A 
related result for the discrete coagulation equation with multiple fragmentation can be found in [14, Section 5]. Although the semigroup techniques we use do not appear to allow for much flexibility with the coagulation terms, we believe, nevertheless, that Theorem 1.2 is of interest in that it establishes, in a much more elementary way, existence and uniqueness results for (1.5) for a larger class of fragmentation kernels than has been dealt with before. In particular, as indicated earlier, previous semigroup investigations $[4,12]$ have assumed that $a$ satisfies $(1.15)$ with $p \leq 1$. We compare our result with other existence/uniqueness theorems for (1.5) in the next section.

Finally we mention that in a recent paper [6], the authors have shown that the number of daughter particles produced by fragmentation remains finite for arbitrary fragmentation rates $a$ and a class of separable size distribution functions $b$ provided only that $\stackrel{\circ}{u \in} X_{0,1}$. This suggests that the results we present here may hold for more general initial conditions when $b$ is a suitable separable function.

\section{Other formulations of the fragmentation equation}

In the work presented here, the fragmentation terms are given by equation (1.2). This is in accordance with the definition used, for example, by Ziff and McGrady [16] and Edwards et al [9]. However, as pointed out in [11], there are other formulations of the fragmentation operator that have attracted attention in recent years. To enable us to compare our results with those obtained in other investigations, first we discuss the relationships between the various formulations.

In many studies, only binary fragmentation has been considered, in which case the (binary) fragmentation operator takes the form

$$
\left(\mathcal{F}_{1} f\right)(x)=-\frac{1}{2} f(x) \int_{0}^{x} F(x-y, y) d y+\int_{x}^{\infty} F(x, y-x) f(y) d y .
$$

The function $F$ is assumed to be symmetric, i.e. $F(x, y)=F(y, x)$ for all $x, y>0$, with $F(x, y)$ representing the rate at which particles of sizes $x$ and $y$ are produced from a fragmenting particle of size $x+y$.

To obtain (2.1) from (1.2), we note first that, in binary fragmentation, the function $b$ must satisfy

$$
n(y)=\int_{0}^{y} b(x \mid y) d x=2 \text { and } b(x \mid y)=b(y-x \mid y),
$$

the latter condition expressing the fact that in a binary fragmentation of a particle of size $y$ the expected number of daughter particles of size $x$ must equal the number of daughter particles of size $y-x,[2,16]$. If we now set

$$
F(x, y):=a(x+y) b(x \mid x+y)
$$


then $F$ is non-negative and symmetric since, by $(2.2)$,

$$
F(y, x)=a(y+x) b(y \mid y+x)=a(x+y) b(x \mid x+y)=F(x, y)
$$

From (2.2), we also obtain

$$
\int_{0}^{x} F(x-y, y) d y=\int_{0}^{x} a(x) b(y \mid x) d y=2 a(x) .
$$

Thus, the identification (2.3) enables (2.1) to be obtained from (1.2). Moreover, on using the symmetry of $F$, we deduce that

$$
\int_{0}^{x} y b(y \mid x) d y=\frac{1}{a(x)} \int_{0}^{x}(x-z) F(z, x-z) d z=2 x-\int_{0}^{x} z b(z \mid x) d z,
$$

which shows that the binary fragmentation is mass conserving.

Similarly, starting with Eq. (2.1), with $F$ symmetric, we obtain (1.2) from (2.3) with $a$ defined by

$$
a(x):=\frac{1}{2} \int_{0}^{x} F(x-y, y) d y .
$$

Then

$$
b(x \mid x+y)=\frac{F(x, y)}{a(x+y)}=\frac{F(y, x)}{a(y+x)}=b(y \mid y+x),
$$

and this gives $b(x \mid y)=b(y-x \mid y)$. From (2.3) and (2.4), we immediately obtain

$$
n(y)=\int_{0}^{y} b(x \mid y) d x=\frac{1}{a(y)} \int_{0}^{y} F(x, y-x) d x=2,
$$

and the same calculation as before yields the mass-conservation condition $\int_{0}^{x} y b(x \mid y) d y=x$.

Having established this correspondence, we can now examine the conditions imposed in some other investigations into binary fragmentation and coagulation. For example, in [8], Dubovskii and Stewart use weak compactness arguments to prove the existence and uniqueness of a non-negative solution for continuous non-negative kernels $k$ and $F$ satisfying

$$
\begin{aligned}
k(x, y) & \leq c(1+x+y) \\
\int_{0}^{x} F(x-y, y) d y & \leq b\left(1+x^{m_{1}}\right) \quad x \in[0, \infty), \\
F\left(x-y^{\prime}, y\right) & \leq b\left(1+x^{m}\right), \quad 0 \leq y \leq y^{\prime} \leq x<\infty
\end{aligned}
$$

where $c, m, m_{1}$ and $b$ are positive constants with $m_{1} \leq 1$. Under an additional constraint, the solution is shown to be mass conserving. From (2.4), we see that (2.6), when expressed in terms of $a$, becomes

$$
a(x) \leq \frac{b}{2}\left(1+x^{m_{1}}\right), \quad 0 \leq m_{1} \leq 1,
$$


which is a more restrictive constraint than (1.15). More recently, Escobedo et al [10] have established the existence of a non-negative weak solution of the CF equation under the following conditions on the binary fragmentation kernel

(i) for each $R \in \mathbb{R}_{+}$there is $F_{R}>0$ such that

$$
F(x, y) \leq F_{R},(x, y) \in S_{R}:=(0, R) \times(0, R),
$$

(ii) there are $R_{0}>0, S_{0} \geq R_{0}$ and $C_{0}>0$ such that

$$
\int_{0}^{R_{0}} F(x-y, y) d y \leq C_{0} \int_{0}^{R_{0}} y F(x-y, y) d y \text { for } x \geq S_{0},
$$

and with $k$ satisfying

$$
k(x, y) \leq c\left((1+x)^{\alpha}(1+y)^{\beta}+(1+x)^{\beta}(1+y)^{\alpha}\right), \quad 0 \leq \alpha \leq \beta \leq 1
$$

In this case, we note first that requirement (i) translates into $a(y+x) b(y \mid y+x)$ being bounded on $S_{R}$. Moreover, by (2.4),

$$
0 \leq a(x) \leq C x
$$

for some $C>0$ as $x \rightarrow 0$. This condition is crucial in the estimates as it enables the action of $A$ in $X_{0}$ to be controlled, close to $x=0$, by the norm of $X_{1}$ (see (1.8)). However, condition (i) is not satisfied by a number of standard fragmentation kernels such as the 'power law' kernels:

$$
a(x)=x^{\alpha}, \alpha \in \mathbb{R}, \quad b(x \mid y)=(\nu+2)\left(\frac{x}{y}\right)^{\nu} y^{-1},-2<\nu \leq 0,
$$

which feature prominently in previous studies, [16]-[18]. These kernels give binary fragmentation for $\nu=0$ but do not satisfy (i) for $0 \leq \alpha<1$. Condition (i) is also not satisfied by fragmentation rates which do not vanish at $x=0$.

We note also that the other assumption placed on $F$, namely (ii), can be given the following physical interpretation in our formulation of fragmentation. Using (2.3) we see that, provided $a(x)>0$ for all $x>0,(2.9)$ reduces to

$$
\int_{0}^{R_{0}} b(y \mid x) d y \leq C_{0} \int_{0}^{R_{0}} y b(y \mid x) d y, \quad x \geq S_{0},
$$

which, from (1.4), means that when sufficiently large particles fragment, the number of daughter particles in a particular size range must be controlled by their total mass in this range. 
Coagulation and multiple fragmentation processes may also be modelled by an equation introduced by Melzak [15], namely

$$
u_{t}(x, t)=-u(x, t) \int_{0}^{x} \frac{y}{x} \gamma(x, y) d y+\int_{x}^{\infty} \gamma(y, x) u(y, t) d y
$$

in which $\gamma(x, y)$ represents the rate at which particles of mass $y$ are produced from fragmenting particles of mass $x$. In this case we recover (1.2) by setting

$$
a(x):=\int_{0}^{x} \frac{y}{x} \gamma(x, y) d y, \text { and } b(x \mid y):=\frac{\gamma(y, x)}{a(y)} .
$$

Existence and uniqueness results for (2.13) coupled with coagulation have been obtained by Laurençot [13] when $k$ takes the form

$$
k(x, y)=r(x) r(y)+\kappa(x, y)
$$

where $r \in C[0, \infty), \kappa \in C([0, \infty) \times[0, \infty))$ and

$$
0 \leq \kappa(x, y)=\kappa(y, x) \leq \operatorname{Dr}(x) r(y) \quad \forall(x, y) \in[1, \infty) \times[1, \infty)
$$

where $D$ is a constant. The function $r$ is the dominant term in the coagulation-fragmentation processes considered in [13] since the kernel $\gamma \in C([0, \infty) \times[0, \infty))$ is assumed to satisfy

$$
\begin{aligned}
\gamma(x, y) & \leq B(1+\max (x, r(x))), x, y \geq 0, \\
\int_{0}^{x} \gamma(x, y) d y & \leq \theta(x) \max (x, r(x)), x \geq 0
\end{aligned}
$$

where $B$ is a positive real number and $\theta:[0, \infty) \rightarrow[0, \infty)$ is such that $\theta(x) \rightarrow 0$ as $x \rightarrow \infty$.

If we consider the case of constant coagulation $k(x, y) \equiv c$ and the 'power law' fragmentation kernels (2.11) (with $\alpha \geq 0$ and $-1<\nu \leq 0$ ), then we obtain $r(x) \equiv \sqrt{c}, \kappa(x, y) \equiv 0$ and

$$
\gamma(x, y):=(\nu+2)\left(\frac{y}{x}\right)^{\nu} x^{\alpha-1}
$$

Clearly $\gamma \notin C([0, \infty) \times[0, \infty))$ unless $\nu=0$ (the binary case) and $\alpha \geq 1$, and for (2.16) to hold, we require $\nu=0$ and $1 \leq \alpha \leq 2$. Moreover,

$$
\int_{0}^{x} \gamma(x, y) d y=\left(\frac{\nu+2}{\nu+1}\right) x^{\alpha}
$$

and so condition (2.17) is not satisfied for any $\nu$, when $\alpha \geq 1$. Consequently, the case of constant coagulation and power law multiple fragmentation cannot be treated using the results of [13]. 


\section{Controlling the number of particles in fragmentation}

In this section we consider only the fragmentation part of Equation (1.1), namely

$$
u_{t}(x, t)=-a(x) u(x, t)+\int_{x}^{\infty} b(x \mid y) a(y) u(y, t) d y
$$

where $a$ and $b$ are assumed to satisfy conditions (A.1)-(A.3). By $\mathcal{A}$ and $\mathcal{B}$ we denote the expressions $[\mathcal{A} f](x)=-a(x) f(x)$ and $[\mathcal{B} f](x)=\int_{x}^{\infty} b(x \mid y) a(y) f(y) d y$, respectively, defined for measurable functions $f$ on $\mathbb{R}_{+}$for which $[\mathcal{A} f](x)$ and $[\mathcal{B} f](x)$ are finite almost everywhere on $\mathbb{R}_{+}$. In keeping with (1.7), let $A:=\left.\mathcal{A}\right|_{D(A)}$ where $D(A)=\left\{f \in X_{1}: a f \in X_{1}\right\}$. It is well known, [3, Chapter 8], that $B:=\left.\mathcal{B}\right|_{D(A)}$ is a well-defined positive operator. Also, as mentioned earlier, the closure $G=\overline{A+B}$ generates a substochastic semigroup $\left\{S_{G}(t)\right\}_{t \geq 0}$ on $X_{1}$; see [3, Theorem 8.5].

For any positive function $\gamma$ on $\mathbb{R}_{+}$, we define

$$
X^{\gamma}:=\left\{f \in X_{1}: \gamma f \in X_{1}\right\}
$$

with the graph norm

$$
\|f\|_{[\gamma]}:=\int_{0}^{\infty}|f(x)| x d x+\int_{0}^{\infty}|f(x)| \gamma(x) x d x .
$$

By $A_{\gamma}$ we denote the part of $A$ in $X^{\gamma}$ with

$$
D\left(A_{\gamma}\right):=\left\{f \in X^{\gamma}: \text { af } \in X^{\gamma}\right\}
$$

We also define

$$
B_{\gamma}:=\left.B\right|_{D\left(A_{\gamma}\right)}
$$

below we shall see that $B_{\gamma}$ is well defined; that is, $D\left(A_{\gamma}\right) \subset D(B)$.

Proposition 3.1 Under the above assumptions and notation, $X^{\gamma}$ is invariant under $\left\{S_{G}(t)\right\}_{t \geq 0}$ provided that $\gamma$ is non-decreasing on $\mathbb{R}_{+}$.

Proof. We note that $\gamma$ is necessarily bounded at 0 . Consider $0 \leq f \in D\left(A_{\gamma}\right)$. Then $x a(x) f(x)$ and $x a(x) \gamma(x) f(x)$ are integrable. Further, by (A.2) and the monotonicity of $\gamma$,

$$
\begin{aligned}
& \int_{0}^{\infty}\left(\int_{x}^{\infty} a(y) b(x \mid y) f(y) d y\right)(x+x \gamma(x)) d x=\int_{0}^{\infty} a(y) f(y)\left(\int_{0}^{y}(x+x \gamma(x)) b(x \mid y) d x\right) d y \\
& \leq \int_{0}^{\infty} a(y) f(y)(y+y \gamma(y)) d y<\infty .
\end{aligned}
$$


Thus, using the fact that the terms of the sum below can be integrated separately if $0 \leq f \in D\left(A_{\gamma}\right)$, we can deduce from (3.2) that

$$
\int_{0}^{\infty}\left(-a(x) f(x)+\int_{x}^{\infty} a(y) b(x \mid y) f(y) d y\right)(x+x \gamma(x)) d x \leq 0 .
$$

Thus, there is an extension $G_{\gamma}$ of $A_{\gamma}+B_{\gamma}$ that generates a positive semigroup of contractions, $\left\{S_{G_{\gamma}}(t)\right\}_{t \geq 0}$, on $X^{\gamma}$. Following the construction technique of [3, Theorem 5.2], we find that the resolvent of the generator is obtained as the monotonic limit of resolvents of $A+r B$ restricted to $X^{\gamma}$ as $r \rightarrow 1^{-}$. Since the lattice structure in $X_{1}$ is the same as in $X^{\gamma}$ (and also the norm in $X^{\gamma}$ is stronger than in $X_{1}$ ), we conclude that, for each $t \geq 0, S_{G_{\gamma}}(t)$ is a restriction of $S_{G}(t)$.

The ideas contained in the proof of Proposition 3.1 can also be used to prove Theorem 1.1.

\section{Proof of Theorem 1.1.}

Let $a$ be non-decreasing on $[M, \infty)$ for some $M>0$ and set $a(x)=a_{1}(x)+a_{2}(x)$ where

$$
a_{1}(x):= \begin{cases}a(x) & \text { for } x \in[0, M] \\ 0 & \text { elsewhere }\end{cases}
$$

and

$$
a_{2}(x):= \begin{cases}0 & \text { for } x \in[0, M], \\ a(x) & \text { elsewhere. }\end{cases}
$$

Then (3.1) can be expressed as an abstract differential equation of the form

$$
u_{t}=A_{2, a} u+B_{2, a} u+A_{1, a} u+B_{1, a} u, t>0,
$$

where, for $i=1,2$,

$$
\begin{aligned}
& \left(A_{i, a} f\right)(x):=-a_{i}(x) f(x) \\
& \left(B_{i, a} f\right)(x):=\int_{x}^{\infty} b(x \mid y) a_{i}(y) f(y) d y .
\end{aligned}
$$

In case (a) of Theorem 1.1, we take

$$
D\left(A_{i, a}\right):=\left\{f \in X^{a}: a_{i} f \in X^{a}\right\}, \quad D\left(B_{i, a}\right):=D\left(A_{i, a}\right),
$$

and in case (b)

$$
D\left(A_{i, a}\right):=\left\{f \in \mathcal{X}^{a}: a_{i} f \in \mathcal{X}^{a}\right\}, \quad D\left(B_{i, a}\right):=D\left(A_{i, a}\right),
$$

with $\mathcal{X}^{a}=X_{0,1} \cap D(A)$ equipped with the norm

$$
\|f\|_{\mathcal{X}^{a}}=\int_{0}^{\infty}|f(x)|(1+x+x a(x)) d x .
$$


Let us continue with the proof of case (a). If we now consider $C_{a}:=A_{1, a}+B_{1, a}$ on $X^{a}$, then clearly $A_{1, a} \in B\left(X^{a}\right)$. Also,

$$
\begin{aligned}
\left\|B_{1, a} f\right\|_{[a]} \leq & \int_{0}^{\infty}\left(\int_{x}^{\infty} a_{1}(y) b(x \mid y)|f(y)| d y\right)(x+x a(x)) d x \\
= & \int_{0}^{\infty} a_{1}(y)|f(y)|\left(\int_{0}^{y} b(x \mid y)(x+x a(x)) d x\right) d y \leq\left(1+a_{M}\right) \int_{0}^{M} a(y)|f(y)| y d y \leq\left(1+a_{M}\right)\|f\|_{[a]},
\end{aligned}
$$

where $a_{M}=\operatorname{ess}_{\sup _{x \in[0, M]}} a(x)$. Consequently, $C_{a} \in B\left(X^{a}\right)$.

It is now convenient to work with the norm $\|\cdot\|_{\left[a_{2}\right]}$ on $X^{a}$, where

$$
\|f\|_{\left[a_{2}\right]}:=\int_{0}^{\infty}|f(x)|\left(x+x a_{2}(x)\right) d x, \quad f \in X^{a} .
$$

Note that $\|\cdot\|_{[a]}$ and $\|\cdot\|_{\left[a_{2}\right]}$ are equivalent norms on $X^{a}$. Indeed

$$
\begin{aligned}
\|f\|_{\left[a_{2}\right]} & \leq\|f\|_{[a]}:=\int_{0}^{\infty}|f(x)|\left(x+x a_{1}(x)+x a_{2}(x)\right) d x \\
& \leq \int_{0}^{\infty}|f(x)|\left(x\left(1+a_{M}\right)+x a_{2}(x)\right) d x \leq\left(1+a_{M}\right)\|f\|_{\left[a_{2}\right]} .
\end{aligned}
$$

Let $0 \leq f \in D\left(A_{2, a}\right)$ so that $x a_{2}(x) f(x)$ and $x\left(a_{2}(x)\right)^{2} f(x)$ are both integrable. Then, arguing as in Proposition 3.1 (with $a$ and $\gamma$ both replaced by $a_{2}$ ), we obtain

$$
\int_{0}^{\infty}\left(-a_{2}(x) f(x)+\int_{x}^{\infty} a_{2}(y) b(x \mid y) f(y) d y\right)\left(x+x a_{2}(x)\right) d x \leq 0 .
$$

Thus, there is an extension $G_{2, a}$ of $A_{2, a}+B_{2, a}$ generating a positive semigroup of contractions, $\left\{S_{G_{2, a}}(t)\right\}_{t \geq 0}$, on $X^{a}$. Moreover, the fact that the operator $C_{a}$ is bounded on $X^{a}$ means that $G_{a}:=G_{2, a}+C_{a}$ also generates a semigroup on $X^{a}$. We note that taking $a \equiv 1$, we have $X^{1}=X_{1}$ and replacing $a$ by 1 in the above notation we recover the original operators acting in $X_{1}$, e.g., $G_{2,1}$ is the extension of $A_{2,1}+B_{2,1}$, defined as in (3.7), which generates a semigroup $\left\{S_{G_{2,1}}(t)\right\}_{t \geq 0}$ on $X_{1}$. As in the proof of Proposition 3.1, we see that $\left\{S_{G_{2, a}}(t)\right\}_{t \geq 0}$ must be a restriction of $\left\{S_{G_{2,1}}(t)\right\}_{t \geq 0}$. Since $C_{1}=A_{1,1}+B_{1,1}$ is also bounded in $X_{1}$ we deduce that the semigroup $\left\{S_{G_{a}}(t)\right\}_{t \geq 0}$ is a restriction of $\left\{S_{G_{1}}(t)\right\}_{t \geq 0}$ generated by $G_{1}=G_{2,1}+C_{1}$. Now, since $a$ and $a_{2}$ are bounded as $x \rightarrow 0$, by [3, Theorem 8.5], $G$ and $G_{2,1}$ are closures of $A+B$ and $A_{2,1}+B_{2,1}$, respectively. Hence, $G=G_{1}$ and $\left\{S_{G_{a}}(t)\right\}_{t \geq 0}$ is a restriction of $\left\{S_{G}(t)\right\}_{t \geq 0}$.

Let us return to the proof of the case (b). As in the case (a), $\|f\|_{\mathcal{X}^{a}}$ is equivalent to

$$
\|f\|_{\mathcal{X}^{a_{2}}}=\int_{0}^{\infty}|f(x)|\left(1+x+x a_{2}(x)\right) d x
$$


and we can prove that that $C_{a}:=A_{1, a}+B_{1, a}$ is a bounded linear operator on $\mathcal{X}^{a}$. From now on we shall work with the norm defined by (3.9).

Let $0 \leq f \in D\left(A_{2, a}\right)$ so that $a_{2}(x)(1+x+x a(x)) f(x)$ is integrable, yielding integrability of each component. We combine (3.8) with

$$
\begin{aligned}
& \int_{0}^{\infty}\left(-a_{2}(x) f(x)+\int_{x}^{\infty} a_{2}(y) b(x \mid y) f(y) d y\right) d x=-\int_{0}^{\infty} a_{2}(x) f(x) d x+\int_{0}^{\infty} a_{2}(y) f(y) n(y) d y \\
& =\int_{1}^{\infty} a_{2}(y) f(y)(n(y)-1) d y \leq \int_{1}^{\infty} a_{2}(y) f(y)\left(c_{2} y+c_{1}-1\right) d y \leq L \int_{1}^{\infty} a_{2}(y) f(y) y d y \\
& \leq L \int_{0}^{\infty} a_{2}(y) f(y) y d y \leq L\|f\|_{\mathcal{X}^{a_{2}}},
\end{aligned}
$$

for some constant $L$, to claim that

$$
\int_{0}^{\infty}\left(\left(A_{2} f\right)(x)+\left(B_{2} f\right)(x)\right)\left(1+x+x a_{2}(x)\right) d x \leq L\|f\|_{\mathcal{X}^{a_{2}}}
$$

for any $0 \leq f \in D\left(A_{2, a}\right)$ which gives the existence of a positive semigroup $\left\{S^{\prime}(t)\right\}_{t \geq 0}$ on $\mathcal{X}^{a}$ satisfying

$$
\left\|S^{\prime}(t) f\right\|_{\mathcal{X}^{a_{2}}} \leq e^{L t}\|f\|_{\mathcal{X}^{a_{2}}} .
$$

Since the operator $C_{a}$ is bounded on $\mathcal{X}^{a}$, we obtain the existence of solutions to the full equation with the above estimate but possibly different $L$ (at worst $L+\|C\|_{\mathcal{X}^{a_{2}}}$ ). As in case (a), this semigroup must be a restriction of $\left\{S_{G}(t)\right\}_{t \geq 0}$ to $\mathcal{X}^{a}$.

Next we prove a variant of part (b) of Theorem 1.1 which plays an important role in the analysis of the combined coagulation-fragmentation. Although the following result gives a weaker control of the invariance space of $\left\{S_{G}(t)\right\}_{t \geq 0}$ than that provided by Theorem 1.1(b), it has the advantage that less restrictive conditions are required on the functions $a$ and $n$.

Theorem 3.2 Let $a$ and $b$ satisfy (A.1)-(A.3) and suppose there is a non-decreasing function $\omega$ such that $a(x) \leq \omega(x)$ for a.a. $x \in \mathbb{R}_{+}$. Further, assume that condition (1.14) holds. Then $\left\{S_{G}(t)\right\}_{t \geq 0}$ leaves $\mathcal{X}_{\omega}:=L_{1}\left(\mathbb{R}_{+},\left(1+x^{r} \omega(x)\right) d x\right)$ invariant.

Proof. First we observe that $\omega$, being monotonic, is necessarily measurable. Let $X_{\omega}$ be equipped with the natural norm

$$
\|f\|_{\omega}=\int_{0}^{\infty}|f(x)|\left(1+x^{r} \omega(x)\right) d x
$$


Then $X_{\omega} \hookrightarrow X_{1}$ since $\omega$ is non-decreasing and not identically zero (as $a$ is not identically zero). Denote by $A_{\omega}$ the part of $A$ in $X_{\omega}$, so that, for $0 \leq f \in D\left(A_{\omega}\right), a(x)\left(1+x^{r} \omega(x)\right) f(x)$ is integrable yielding, of course, integrability of each component. As before, $B_{\omega}=\left.\mathcal{B}\right|_{D\left(A_{\omega}\right)}$. Further, since $x \rightarrow x^{r-1} \omega(x)$ is also non-decreasing, then, as in the proof of Proposition 3.1, we obtain, for $0 \leq f \in D\left(A_{\omega}\right)$,

$$
\begin{aligned}
& \int_{0}^{\infty}\left(\int_{x}^{\infty} a(y) b(x \mid y) f(y) d y\right) x^{r} \omega(x) d x=\int_{0}^{\infty} f(y) u(y)\left(\int_{0}^{y} x^{r} \omega(x) b(x \mid y) d x\right) d y \\
& \leq \int_{0}^{\infty} a(y) f(y) y^{r-1} \omega(y)\left(\int_{0}^{y} x b(x \mid y) d x\right) d y=\int_{0}^{\infty} a(y) f(y) y^{r} \omega(y) d y<\infty
\end{aligned}
$$

Next, as in the proof of Theorem 1.1 (b), we have

$$
\begin{aligned}
& \int_{0}^{\infty}\left(-a(x) f(x)+\int_{x}^{\infty} a(y) b(x \mid y) f(y) d y\right) d x=-\int_{0}^{\infty} a(x) f(x) d x+\int_{0}^{\infty}\left(\int_{x}^{\infty} a(y) b(x \mid y) f(y) d y\right) d x \\
& =-\int_{0}^{\infty} a(x) f(x) d x+\int_{0}^{\infty} a(y) f(y)\left(\int_{0}^{y} b(x \mid y) d x\right) d y=-\int_{0}^{\infty} a(x) f(x) d x+\int_{0}^{\infty} a(y) f(y) n(y) d y \\
& =\int_{0}^{\infty} a(y) f(y)(n(y)-1) d y \leq \int_{0}^{\infty} \omega(y)\left(c_{2} y^{r}+c_{1}-1\right) f(y) d y \\
& \leq c_{2} \int_{0}^{\infty} \omega(y) y^{r} f(y) d y+\left(c_{1}-1\right)\left(\int_{0}^{1} \omega(y) f(y) d y+\int_{1}^{\infty} \omega(y) f(y) d y\right) \\
& \leq\left(c_{2}+c_{1}-1\right) \int_{0}^{\infty} \omega(y) y^{r} f(y) d y+\left(c_{1}-1\right) l \int_{0}^{\infty} f(y) d y \leq L\|f\|_{\omega},
\end{aligned}
$$

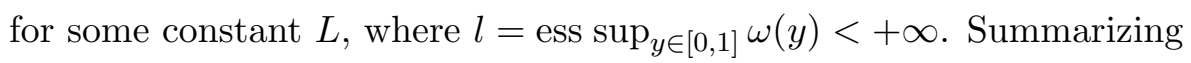

$$
\int_{0}^{\infty}\left(\left(A_{\omega} f\right)(x)+\left(B_{\omega} f(x)\right)\left(1+x^{r} \omega(x)\right) d x \leq L\|f\|_{\omega},\right.
$$

for any $0 \leq f \in D\left(A_{\omega}\right)$ which, by the argument of [3, Proposition 9.29], gives the existence of a positive semigroup $\left\{S_{G_{\omega}}(t)\right\}_{t \geq 0}$ on $X_{\omega}$ satisfying

$$
\left\|S_{G_{\omega}}(t) f\right\|_{\omega} \leq e^{L t}\|f\|_{\omega} .
$$

As in the proof of Proposition 3.1, this semigroup must be a restriction of $\left\{S_{G}(t)\right\}_{t \geq 0}$ to $X_{\omega}$.

Remark 3.3 We note that for any $a \in L_{\infty, l o c}([0, \infty))$ we can easily obtain a function $\omega$ satisfying the conditions of the above theorem by defining

$$
\omega(x)=\operatorname{ess}_{\sup _{y \in[0, x]}} a(y) .
$$


Remark 3.4 A consequence of Theorem 3.2 is that in any (polynomially bounded) fragmentation process starting with an initial distribution which is sufficiently small for large masses, the total number of particles remains finite for any finite time.

\section{The coagulation-fragmentation equation}

Let us now return to the full fragmentation-coagulation equation (1.1). In addition to the standard assumptions on the fragmentation and coagulation coefficients, namely (A.1)-(A.4), we also assume that $b$ satisfies $(1.14)$ and that constants $C>0$ and $p \in(1, \infty)$ exist such that

$$
a(x) \leq C\left(1+x^{p}\right), \quad x \geq 0 .
$$

We note that the case $p \leq 1$ has been studied in [4] and including it here would create some technical inconveniences. We introduce the expression $\mathcal{N}$ defined by

$$
\mathcal{N}[f, g](x):=\frac{1}{2} \int_{0}^{x} k(x-y, y) f(x-y) g(y) d y-f(x) \int_{0}^{\infty} k(x, y) g(y) d y, \quad x>0
$$

and denote $\|k\|_{L_{\infty}}=k_{0}$.

We shall study (1.1) in the space $X_{\omega}$ introduced in Theorem 3.2. Then $\omega$ can be any non-decreasing function satisfying $\omega(x) \geq C x^{r}\left(1+x^{p}\right)$. Since $x^{r}\left(1+x^{p}\right) \leq \tilde{C}\left(1+x^{\beta}\right)$ for any $\beta \geq m=p+r$ and a constant $\tilde{C}$, in what follows we shall work in $X_{\omega}=X_{0, \beta}$ with $\beta \geq m=r+p$. Accordingly, $A_{0, \beta}, B_{0, \beta}, G_{0, \beta},\left\{S_{0, \beta}(t)\right\}_{t \geq 0}$ denote, respectively, the parts of $A, B, G$ and $\left\{S_{G}(t)\right\}_{t \geq 0}$ in $X_{0, \beta}$.

Proposition 4.1 The expression $\mathcal{N}$ restricted to $X_{0, \beta} \times X_{0, \beta}$ is an $X_{0, \beta}$-valued, continuous and bilinear operator. Thus, denoting $N_{0, \beta} f:=\mathcal{N}[f, f]$ for $u \in X_{0, \beta}, N_{0, \beta}$ is continuously Fréchet differentiable at any point $f \in X_{0, \beta}$ and therefore locally Lipschitz on $X_{0, \beta}$. Consequently, for $0 \leq \stackrel{\circ}{u} \in X_{0, \beta}$ there exists a unique non-negative mild solution, and for any $0 \leq \stackrel{\circ}{u} \in D\left(G_{0, \beta}\right)$ there exists a unique non-negative strict solution, $u(t)=U_{0, \beta}(t, \stackrel{\circ}{u})$ of

$$
u_{t}(t)=G_{0, \beta} u(t)+N_{0, \beta} u(t)
$$

defined on its maximal interval of existence $[0, t(\stackrel{\circ}{u}))$, where $t(\stackrel{\circ}{u})>0$.

Proof. For future use, we consider the integral

$$
\int_{0}^{\infty} \phi(x)[\mathcal{N}(f, g)](x) d x
$$


where $\phi, f, g$ are appropriately restricted functions. Then, routine calculations show that

$$
\begin{aligned}
& \int_{0}^{\infty} \phi(x)[\mathcal{N}(f, g)](x) d x \\
= & \frac{1}{2} \int_{0}^{\infty} \int_{0}^{\infty} \phi(x+y) k(x, y) f(x) g(y) d x d y-\int_{0}^{\infty} \int_{0}^{\infty} \phi(x) k(x, y) f(x) g(y) d x d y,
\end{aligned}
$$

and, in particular, putting $g=f$ and using the symmetry of $k$,

$$
\int_{0}^{\infty} \phi(x)[\mathcal{N}(f, f)](x) d x=\frac{1}{2} \int_{0}^{\infty} \int_{0}^{\infty} \tilde{\phi}(x, y) k(x, y) f(x) f(y) d x d y
$$

where

$$
\tilde{\phi}(x, y)=\phi(x+y)-\phi(x)-\phi(y)
$$

Thus, using the inequality

$$
(x+y)^{\beta} \leq 2^{\beta}\left(x^{\beta}+y^{\beta}\right), \quad \beta \geq 0,
$$

we establish that

$$
\int_{0}^{\infty}\left(1+x^{\beta}\right)|[\mathcal{N}(f, g)](x)| d x \leq K_{1}\left(\|f\|_{0}\|g\|_{0}+\|f\|_{\beta}\|g\|_{0}+\|f\|_{0}\|g\|_{\beta}\right) \leq K\|f\|_{0, \beta}\|g\|_{0, \beta}
$$

for some constants $K_{1}$ and $K$. Hence, $N_{0, \beta}$ is a bounded bilinear form and the remaining part of the proof follows as in [5].

In what follows we shall need a refinement of (4.5). One could use a modification of the result for the discrete C-F equation given in [7, Lemma 2.3]. However, a simpler estimate is possible.

Lemma 4.2 For any $x, y \in \mathbb{R}_{+}$and $\beta \geq 1$ we have

$$
0 \leq(x+y)^{\beta}-x^{\beta}-y^{\beta} \leq\left(2^{\beta}-1\right)\left(x y^{\beta-1}+y x^{\beta-1}\right) .
$$

Proof. The first inequality is obvious. We can take $\beta>1$. Then, the function $(1+w)^{\beta}$ is concave and thus its graph lies below the line passing through $(0,1)$ and $\left(1,2^{\beta}\right)$ as long as $0 \leq w \leq 1$. In other words,

$$
(1+w)^{\beta} \leq\left(2^{\beta}-1\right) w+1, \quad 0 \leq w \leq 1
$$

Then, on setting $w=y / x$ with $0<y \leq x$ we obtain

$$
(x+y)^{\beta} \leq x^{\beta}+\left(2^{\beta}-1\right) y x^{\beta-1}
$$

and, by symmetry, for any $x, y>0$,

$$
(x+y)^{\beta} \leq x^{\beta}+y^{\beta}+\left(2^{\beta}-1\right)\left(y^{\beta-1} x+y x^{\beta-1}\right) .
$$




\section{Proof of Theorem 1.2}

In view of Proposition 4.1, we only have to prove that any solution $t \rightarrow U_{0, m}(t, \stackrel{\circ}{u})$, with $0 \leq \stackrel{\circ}{u} \in$ $X_{0, m}$, is global in time. First we observe that if $0 \leq \stackrel{\circ}{u} \in X_{0, \beta}, \beta=p+m$, then $t \rightarrow U_{0, p+m}(t) \stackrel{\circ}{u}$ is a mild solution to (4.2) in $X_{0, \beta}$. However, we have $D\left(A_{0, m}\right)=\left\{f \in X_{0, m}:\right.$ af $\left.\in X_{0, m}\right\}$ and if $a(x) \leq C\left(1+x^{p}\right)$, then $X_{0, p+m} \subset D\left(A_{0, m}\right) \subset G_{0, m}$ and for such $\stackrel{\circ}{u}, t \rightarrow U_{0, p+m}(t) \stackrel{\circ}{u}=U_{0, m}(t) \stackrel{\circ}{u}$ is a strict solution to $(4.2)$ in $X_{0, m}$. Define this solution by $u(t)=u(\cdot, t)$. Then we have

$$
\int_{0}^{\infty} x^{l} a(x) u(x, t) d x \leq C \int_{0}^{\infty} x^{l}\left(1+x^{p}\right) u(x, t) d x<+\infty
$$

as long as $l \leq m$. Because of (4.6), Equation (1.1) can be multiplied by $x^{l}$ and integrated termwise for any $l \leq m$. Denoting $M_{l}(t)=\int_{0}^{\infty} x^{l} u(x, t) d x$ we have

$$
\begin{aligned}
M_{0}^{\prime} & =\int_{0}^{\infty} a(x)(n(x)-1) u(x, t) d x-\frac{1}{2} \int_{0}^{\infty} \int_{0}^{\infty} k(x, y) u(x, t) u(y, t) d x d y, \\
M_{1}^{\prime} & =0 .
\end{aligned}
$$

Therefore $M_{1}(t)=$ const $=M_{1}(0)$ and we have the estimate

$$
M_{0}^{\prime} \leq L_{1} M_{0}+L_{2} M_{m}
$$

for some constants $L_{1}, L_{2}$ depending only on the coefficients but not on the initial value for $u$.

To find an estimate for $M_{m}$, first we observe that

$$
\begin{aligned}
& \int_{0}^{\infty} x^{m}\left(\int_{x}^{\infty} a(y) b(x \mid y) u(y, t) d y\right) d x=\int_{0}^{\infty} a(y) u(y, t)\left(\int_{0}^{y} x^{m} b(x \mid y) d x\right) d y \\
& \leq \int_{0}^{\infty} a(y) u(y, t) y^{m-1}\left(\int_{0}^{y} x b(x \mid y) d x\right) d y=\int_{0}^{\infty} a(y) u(y, t) y^{m} d y .
\end{aligned}
$$

Thus, with the help of (4.4), Lemma 4.2 and the estimate $x^{\beta-1} \leq x+x^{\beta}$ for $x>0, \beta \geq 2$,

$$
M_{m}^{\prime} \leq \frac{1}{2} \int_{0}^{\infty} \int_{0}^{\infty}\left((x+y)^{m}-x^{m}-y^{m}\right) k(x, y) u(x, t) u(y, t) d x d y \leq K\left(M_{1}^{2}+M_{1} M_{m}\right),
$$

for some constant $K$ which is independent of $\stackrel{\circ}{u}$. Integrating the last inequality, we obtain

$$
0 \leq M_{m}(t) \leq e^{K M_{1} t} M_{m}(0)+M_{1}\left(e^{K M_{1} t}-1\right)
$$

and, inserting the latter into (4.8) and dropping the negative term, we have

$$
M_{0}^{\prime} \leq L_{1} M_{0}+L_{2}\left(M_{m}(0)+M_{1}\right) e^{K M_{1} t} .
$$


Upon integration this gives

$$
M_{0}(t) \leq e^{L_{1} t} M_{0}(0)+\frac{L_{2}\left(M_{m}(0)+M_{1}\right)}{\left(K M_{1}-L_{1}\right)}\left(e^{K M_{1} t}-e^{L_{1} t}\right)
$$

where, without loss of generality, $K$ and $L_{1}$ are chosen so that $K M_{1}-L_{1}>0$. Since

$$
\left\|U_{0, m}(t) \stackrel{\circ}{u}\right\|_{0, m}=M_{0}(t)+M_{m}(t),
$$

we see that $U_{0, m}(t) \stackrel{\circ}{u}$ is defined globally in time for any $\stackrel{\circ}{u} \in X_{0, p+m}$. However, $X_{0, p+m}$ is dense in $X_{0, m}, U_{0, m}(t)$ depends continuously on the initial condition (on a common interval of existence) by the Gronwall inequality, and it is clear that the right-hand sides of (4.9) and (4.10) depend continuously on the zeroth and $m$-th moments of the initial condition. Thus, these inequalities can be extended to the moments of a solution originating from any $\stackrel{\circ}{u} \in X_{0, m}$. Hence, any solution of (1.1) in $X_{0, m}$ is global in time.

\section{References}

[1] J. Banasiak, On a non-uniqueness in fragmentation models, Math. Methods Appl. Sci, 25 (2002), 541-556.

[2] J. Banasiak, Conservative and shattering solutions for some classes of fragmentation equations, Math. Models Methods Appl. Sci., 14(4), 2004, 483-501.

[3] J. Banasiak, L. Arlotti, Positive perturbations of semigroups with applications, Springer, London, 2006.

[4] J. Banasiak, W. Lamb, On a coagulation and fragmentation equation with mass loss, Proc. Roy. Soc. Edinburgh, 136A, (2006), 1157-1173.

[5] J. Banasiak, W. Lamb, Coagulation, fragmentation and growth processes in a size structured population, Discrete and Continuous Dynamical Systems-B, 11(3), (2009), 563-585, doi:10.3934/dcdsb.2009.11.563.

[6] J. Banasiak, S. C. Oukoumi Noutchie, Controlling number of particles in fragmentation equations, Physica D, 239, (2010), 1422-1435.

[7] J. Carr, Asymptotic behaviour of solutions to the coagulation-fragmentation equations. I. The strong fragmentation case, Proc. Roy. Soc. Edinburgh, 121A, (1992), 231-244. 
[8] P. B. Dubovskii and I. W. Stewart, Existence, uniqueness and mass conservation for the coagulation-fragmentation equation, Math. Meth. Appl. Sci., 19 (1996), 571-591.

[9] B. F. Edwards, M. Cai, H. Han, Rate equation and scaling for fragmentation with mass loss, Phys. Rev. A, 41, (1990), 5755-5757.

[10] M. Escobedo, Ph. Laurençot, S. Mischler, B. Perthame, Gelation and mass conservation in coagulation-fragmentation models, J. Differential Equations, 195(1), (2003), 143-174.

[11] W. Lamb, A.C. McBride, On a continuous coagulation and fragmentation equation with a singular fragmentation kernel. In Evolution Equations G.R. Goldstein, R. Nagel and S. Romanelli (editors), Lecture Notes in Pure and Applied Mathematics 234, Marcel Dekker, New York (2003), 583-599.

[12] W. Lamb, Existence and uniqueness results for the continuous coagulation and fragmentation equation, Math. Methods Appl. Sci., 27 (2004), 703-721.

[13] P. Laurençot, On a class of continuous coagulation-fragmentation equations, J. Differential Equations, 167(2), (2000), 245-274.

[14] P. Laurençot, The discrete coagulation equations with multiple fragmentation, Proc. Edinburgh Math. Soc., 45 (2002), 67-82.

[15] Z. A. Melzak, A scalar transport equation, Trans. Amer. Math. Soc.,85 (1957), 547-560.

[16] E. D. McGrady, R. M. Ziff, "Shattering" transition in fragmentation, Phys. Rev. Lett., 58(9), (1987), 892-895.

[17] R. M. Ziff, E. D. McGrady, The kinetics of cluster fragmentation and depolymerization, J. Phys. A: Math. Gen. 18, (1985), 3027-3037.

[18] R. M. Ziff, E. D. McGrady, Kinetics of polymer degradation, Macromolecules, 19 (1986), $2513-2519$. 